\title{
Treino Cognitivo para Idosos: Uma Revisão Sistemática dos Estudos Nacionais
}

\author{
Mariana Teles Santos - Universidade Federal da Babia, Salvador, Brasil \\ Carmen Flores-Mendoza - Universidade Federal de Minas Gerais, Belo Horizonte, Brasil
}

\begin{abstract}
Resumo
Este artigo de revisão sistemática sobre treino cognitivo para idosos saudáveis objetivou analisar 23 estudos nacionais em seus aspectos metodológicos e quanto às evidências de eficácia das intervenções. Os estudos foram publicados entre os anos 2000 e 2015. Uma busca foi feita nas bases SciELO, PubMed e PsycInfo utilizando-se os termos (português e inglês): "treino cognitivo", "estimulação cognitiva", "envelhecimento", "idoso" e "brasileiro". Em uma segunda etapa, as referências dos artigos encontrados foram consultadas para identificar quaisquer estudos pertinentes adicionais. A quase totalidade dos estudos utilizou a modalidade coletiva de intervenção $(n=20)$. O número de sessões com os participantes variou de 2 a 48 encontros. Em relação às habilidades cognitivas que foram alvo de intervenção, $60,8 \%$ dos estudos $(n=14)$ tiveram como foco o treino de memória episódica. Como medidas cognitivas, $90,4 \%$ dos estudos $(n=20)$ incluíram escalas de domínio comum (não restritas a psicólogos). No tocante aos resultados, $47,6 \%$ dos estudos $(n=10)$ identificaram efeitos de treino para pelo menos uma medida cognitiva utilizada. Conclui-se que o panorama nacional em treino cognitivo para idosos destaca-se por uma tendência a intervenções coletivas e voltadas à estimulação da memória episódica. Discute-se a importância de um avanço na área em relação à ampliação das pesquisas e à testagem da eficácia das intervenções padronizadas para o contexto brasileiro.
\end{abstract}

Palavras-chave: inteligência, intervenção, revisão

Cognitive training for elderly: a systematic review of Brazilian research

\begin{abstract}
This systematic review about cognitive training for healthy elderly adults aimed to analyze 23 Brazilian studies regarding their methodological aspects and the evidence of effectiveness of interventions. The studies were published between 2000 and 2015. A search was performed in Scielo, PubMed and PsycInfo databases using the terms (Portuguese and English): cognitive training, cognitive stimulation, aging, elderly, and Brazilian. In a second step, the references of retrieved articles were consulted to identify any additional relevant studies. Almost all studies used the group intervention modality $(n=20)$. The number of sessions with participants ranged from 2 to 48 meetings. Regarding the cognitive skills that were targeted for intervention, $60.8 \%$ of the studies $(n=14)$ were focused on episodic memory training. For cognitive measures, $90.4 \%$ of the studies $(n=20)$ included common domain scales (not restricted to psychologists). Regarding the results, $47.6 \%$ of studies $(n=10)$ identified training purposes for at least one cognitive measure used. In conclusion, the national scene in cognitive training for older adults stands out for a tendency for collective interventions aimed at stimulating episodic memory. The present article argues for the importance of testing the effectiveness of interventions protocols.
\end{abstract}

Keywords: Intelligence, Intervention, Revision

Entrenamiento cognitivo para ancianos: una revisión sistemática de estudios nacionales

\begin{abstract}
Resumen
Este artículo de revisión sistemática sobre entrenamiento cognitivo para ancianos con buena salud, realizó un análisis de 23 estudios brasilenõs, publicados entre 2000 y 2015, sobre los aspectos metodológicos y evidencias de eficacia de las intervenciones.

Se realizó una búsqueda en las bases Scielo, PubMed y PsycInfo utilizando los términos (portugués e inglés): entrenamiento cognitivo, estimulación cognitiva, envejecimiento, anciano y brasileño. En una segunda etapa, se consultaron las referencias de los artículos encontrados para identificar los estudios pertinentes adicionales. Casi todos los estudios utilizaron la modalidad colectiva de intervención $(\mathrm{n}=20)$. El número de sesiones con los participantes osciló entre 2-48 reuniones. En cuanto a las habilidades cognitivas que fueron objeto de intervención, el 60,8\% de los estudios ( $\mathrm{n}=14$ ) se centraron en el entrenamiento de la memoria episódica. Como medidas cognitivas, $90,4 \%$ de los estudios $(\mathrm{n}=20)$ incluyeron escalas de dominio común (no restringidas a psicólogos) y 47,6\% de los estudios $(n=10)$ identificaron efectos de entrenamiento para por lo menos una medida cognitiva utilizada. Se concluye, que el panorama nacional en entrenamiento cognitivo para ancianos se destaca por una tendencia a intervenciones colectivas y dirigidas a estimulación de la memoria episódica. Se discute la importancia de un avance en el área, con relación a la ampliación de las investigaciones y la comprobación de la eficacia de las intervenciones estandarizadas para el contexto brasileño.

Palabras-clave: Inteligencia, Intervención, Revisión
\end{abstract}

O estudo sobre o envelhecimento cognitivo, especialmente no tocante ao seu curso, vem atraindo crescente investimento de pesquisas que buscam responder até que ponto é possível reverter o declínio

Disponivel em www.scielo.br mental esperado com a idade, assim como o declínio patológico (Ball et al., 2002; Kelly et al., 2014; Valenzuela \& Sachdev, 2009). Um dos caminhos para elucidar tais questões consiste na condução de delineamentos 
experimentais que permitam testar os efeitos de intervenções cognitivas (classificadas no amplo conjunto das intervenções não farmacológicas). Nestas, os mecanismos cognitivos específicos são isolados e treinados de forma a investigar de maneira direta e experimentalmente controlada o grau de plasticidade do funcionamento intelectual durante o envelhecimento (Kramer \& Willis, 2003).

Um dos pressupostos subjacentes às intervenções cognitivas é que a prática de exercícios mentais tem o potencial de melhorar ou, pelo menos, preservar o funcionamento de determinado domínio cognitivo. $\mathrm{Na}$ literatura internacional, as modalidades mais comuns de intervenção cognitiva parecem caminhar para um consenso taxonômico (Bahar-Fuchs, Clare, \& Woods, 2013), delimitando três tipos principais: (a) estimulação mental, que geralmente envolve a realização e a prática repetida de tarefas cognitivas padronizadas; (b) treino cognitivo, que engloba tarefas de estimulação mental, mas avança em termos de esforço de aprendizagem ao focar no ensino de estratégias cognitivas para potencializar os efeitos da intervenção (Bruschet et al., 2003; Clare \& Woods, 2004); e (c) reabilitação cognitiva, conhecida também como abordagem individualizada, em que se atinge, uma de cada vez, deficiências específicas na vida cotidiana, em vez de melhorar o desempenho em tarefas cognitivas particulares (Clare, Woods, Cook, Orrell, \& Spector, 2003; Clare \& Woods, 2004). Essas abordagens são complementares, sendo que a escolha de um tipo particular depende dos objetivos da melhoria ou manutenção cognitiva e do perfil cognitivo da população-alvo (Burschet et al., 2010).

No presente artigo, será apresentada uma revisão sistemática dos estudos de treino cognitivo para idosos saudáveis conduzidos no contexto brasileiro, no que diz respeito aos aspectos estruturais e metodológicos das intervenções, bem como às evidências reportadas de eficácia para aumento do desempenho cognitivo do grupo experimental. As intervenções do tipo estimulação cognitiva e treino cognitivo foram selecionadas para revisão e consideradas sem distinção devido à sua proximidade e ao fato de serem definidas como complementares em trabalhos que buscaram um referencial taxonômico para a área (ex.: Belleville, 2008; BaharFuchs, Clare, \& Woods, 2013; Clare et al., 2003).

Os treinos cognitivos podem assumir diferentes formatos, variando em relação a: 1) modalidade de condução: sessões individuais ou coletivas; 2) habilidades-alvo: intervenção multidomínio - delineada para estimular habilidades cognitivas de diferentes domínios
- ou unimodal - quando as habilidades-alvo fazem parte de um mesmo domínio cognitivo; 3) formato dos estímulos: do tipo lápis e papel ou com tarefas computadorizadas; 4) medidas cognitivas: as medidas podem cobrir as habilidades-alvo da intervenção (aquelas que se pretende intervir) para investigar os efeitos de transferência proximal (near transfer), ou as habilidades não treinadas para investigar os efeitos de transferência distal (far transfer); 5) follow-up: exames de acompanhamento a longo prazo para verificar os efeitos de durabilidade temporal da intervenção.

Esses diversos formatos de intervenção vêm sendo testados quanto ao seu impacto no desempenho cognitivo de idosos e, quando são observados efeitos de ganho, busca-se verificar a duração temporal deles e a generalização para contextos distintos de aprendizagem, como resolução de problemas cotidianos. As investigações sobre o impacto dos treinos começaram a despontar no cenário internacional na década de 1980, no contexto dos projetos Adult Development and Enrichment Project (ADEPT, Baltes \& Willis, 1982) e do Seattle Longitudinal Study (SLS, Schaie \& Willis, 1986). Ambos os projetos envolveram estudos experimentais sobre a reversão do declínio cognitivo relacionado à idade. As intervenções consistiram em cinco horas de ensino de estratégias cognitivas administradas em pequenos grupos no caso do ADEPT, e individualmente para o SLS. Os resultados desses estudos apontaram para ganhos significativos de near transfer para participantes acima dos 60 anos. Os estudos iniciais também investigaram a reversibilidade (total ou parcial) do funcionamento da memória episódica por meio de treino e prática (Baltes \& Linderberg, 1998; Schaie \& Willis, 1986; Willis, Blieszener, \& Baltes, 1981; Willis \& Nesselroade, 1990).

O início desse campo de estudos coincide com o aumento da longevidade média da população nos países desenvolvidos, o que gerou uma nova demanda por serviços de financiamento, suporte e também políticas públicas destinadas à população idosa (Neri, 1993). Tomado em conjunto, as primeiras pesquisas demonstraram que os idosos saudáveis são capazes de melhorar seu nível de desempenho (comparado ao nível anterior à intervenção), quando este é avaliado por medidas cognitivas que mensuram habilidades que foram treinadas. Tais achados foram replicados nas décadas posteriores e, atualmente, os estudos internacionais apresentam importantes avanços metodológicos, configurando-se como uma área bastante heterogênea quanto ao formato e estrutura das intervenções (Ball et al., 2002; Hertzov, Kramer, Wilson, \& Lindenberger, 2009). 
O acúmulo de evidências internacionais permitiu a realização de estudos de meta-análise. Esse tipo de estudo permite integrar resultados de vários estudos independentes, excluindo aqueles que incorporam viés às interpretações. $\mathrm{O}$ primeiro estudo de meta-análise foi registrado na década de 1990 (Verhaeghen, 1992), seguindo-se a ele revisões que focaram em diferentes aspectos, como perfil da amostra e tipos de intervenção (ver alguns dos principais estudos de meta-análise e revisão sistemática internacionais: Belleville, 2008; Gates, Sachdev, Singh, \& Valenzuela, 2011; Law, Barnett, Yau, \& Gray, 2014; Kelly et al., 2014; Martin, Clare, Altsgassen, Cameron, \& Zehnder, 2011; Papp, Stephen, \& Peter, 2009; Reijnders, van Heugten, \& van Boxtel, 2013; Simon, Yokomizo, \& Bottino, 2012). De acordo com os resultados desses estudos internacionais, é possível observar um consenso sobre os efeitos de ganho dos treinos do tipo near transfer e para aspectos funcionais, como resolução de tarefas cotidianas e incremento da autonomia e independência. Contudo, algumas questões centrais ainda permanecem divergindo a área: 1) a transferência de ganhos de intervenção para tarefas e habilidades não treinadas, ou seja, os do tipo far transfer, 2) a manutenção dos efeitos do treino ao longo do tempo (durabilidade temporal); 3) a generalização dos ganhos do treino em contextos de aprendizagem distintos (profissional, acadêmico, social). Tais questões confrontam a hipótese do enriquecimento cognitivo, isto é, seria possível alcançar uma modificação estrutural da cognição durante o envelhecimento? (Hertzov et al., 2009; Salthouse, 2006).

No contexto brasileiro, os estudos sobre treino cognitivo para idosos foram iniciados nos anos 2000 com o estudo do pesquisador Guilherme Wood (Wood, 2000). Esse início tardio em relação às pesquisas internacionais levou a um menor acúmulo de evidências sobre a eficácia dos programas no país e, naturalmente, a um número reduzido de protocolos de intervenção padronizados para o contexto brasileiro. Considerando as discrepâncias em termos de avanços nos cenários internacional e nacional, o presente artigo tem como objetivo apresentar uma revisão sistemática dos ensaios clínicos nacionais em treino cognitivo para idosos saudáveis (sem diagnóstico de quadro demencial), traçando um perfil metodológico dos estudos nacionais e levantando a eficácia reportada das intervenções. O presente estudo localizou apenas um artigo nacional de revisão dessa literatura (Olchik, Mattos, Maineri, Oliveira, \& Yassuda, 2012), porém, com foco em estudos nacionais e internacionais. Na presente revisão, objetiva-se traçar um perfil exclusivo das pesquisas nacionais na área, especialmente em relação ao formato das intervenções brasileiras e seus principais achados.

\section{Método}

Foram pesquisados, entre janeiro de 1990 e abril de 2016, os termos (em português e inglês): treino cognitivo, estimulação cognitiva, envelhecimento, idoso e brasileiro nas bases SciELO, PsycInfo e PubMed. Em uma segunda etapa, as referências bibliográficas foram consultadas para identificar quaisquer estudos pertinentes adicionais. Nessa segunda etapa, foi possível identificar trabalhos de dissertação e tese, disponíveis em bibliotecas universitárias digitais, exceto para Wood (2000), que devido ao ano de publicação, não tem seu trabalho disponível nos acervos digitais.

Como critérios de inclusão para análise dos artigos, utilizou-se: 1) artigos escritos em português ou inglês; 2) estudo de qualquer tipo de treino ou estimulação cognitiva para população de idosos saudáveis (idade superior a 60 anos e sem diagnóstico de quadro demencial) e com comprometimento cognitivo leve; 4) estudos que incluam, pelo menos, avaliações antes e após a intervenção; 5) estudos que utilizem análises estatísticas para embasar as inferências sobre os dados. No tocante aos critérios de exclusão, foram considerados: 1) estudos de reabilitação cognitiva para grupo clínico (quadro demencial). Não foram considerados como critérios de exclusão: a não randomização na distribuição da amostra e a ausência de grupo controle.

Os estudos foram analisados em relação a: a) número de sessões; b) perfil da amostra; c) domínios cognitivos; d) medidas cognitivas utilizadas; e) modalidade de condução (coletiva ou individual); f) eficácia das intervenções; g) exames de acompanhamento a longo prazo; h) origem da intervenção: nacional ou internacional

\section{Resultados}

Ao todo, foram analisados vinte e três estudos nacionais. Para alguns trabalhos de tese/dissertação, não foram identificados artigos com publicação dos resultados, e tais fontes foram, portanto, unicamente analisadas: São elas: 1) Wood (2000); 2) Santos (2010); 3) Lasca (2003). Alguns artigos foram substituídos pela análise da tese/dissertação que os originaram com fins de ampliar o acesso a informações detalhadas sobre as intervenções. Foram eles: 1) Brum (2012); 2) 
Chariglione (2010, 2014); 3) Olchik (2008). A Figura 1 detalha o processo de seleção dos estudos.

$\mathrm{Na}$ Figura 2, é interessante observar que o número de publicações que se enquadra nos critérios deste estudo aumentou gradativamente entre os anos de 2000 e 2015, atingindo um pico no ano de 2010.

Para análise dos resultados, os estudos serão referenciados de acordo com a seguinte numeração atribuída: sessões (Estudo 2) a intervenções de doze encontros (Estudos 3, 10, 15) e até 48 sessões (Estudo 20). É possível observar uma tendência a desenhar intervenções com número de sessões superior a cinco: no total, seis estudos tiveram intervenções com número de sessões inferior a cinco $(28,57 \%)$, e os demais estudos $(71,4 \%)$ conduziram intervenções com número de sessões superior a cinco.
(1) Wood (2000)

(2) Lasca (2003)

(3) Irigaray, Schneider e

Gomes (2004)

(4) Yassuda, Batistoni, Fortes e Neri (2006)

(5) Olchik (2008)

(6) Silva e Yassuda (2009)
(7) Lima-Silva et al. (2010)

(8) Lima-Silva e Yassuda (2010)

(9) Santos (2010)

(10) Irigaray, Filho e Schneider (2010)

(11) Carvalho, Neri e Yassuda (2010)

(12) Chariglione (2010)
(13) Lima-Silva e Yassuda (19) Chariglione (2014) (2011)

(14) Aramaki e Yassuda (20) Oliveira et al. (2014) (2011)

(15) Brum (2012)

(21) Zimmerman, Netto, Amodeo, Ska e Fonseca, (2014)

(16) Dias e Lima (2012)

(22) Tavares, Shimidt e Witter (2015)

(23) Soares, Santana e Rabelo (2015) al. (2012)

(18) Paulo e Yassuda (2012)
A partir da análise dos estudos reportados, é possível observar algumas características que delimitam tendências no campo da pesquisa nacional. Dentre elas, destaca-se o predomínio da modalidade coletiva de intervenção, utilizada em $90,9 \%$ dos estudos $(n=$ 20: estudos 2 a 20 e estudo 23; embora o estudo 22 não informa modalidade de condução) na qual as sessões de treino são conduzidas em grupos que variam em número de participantes, com exceção do estudo de Wood (2000), que conduziu o treino nas duas modalidades. Segundo os autores desses 20 estudos, a opção pela modalidade coletiva justifica-se pelo aumento da motivação e adesão dos participantes ao treino, além de possibilitar a troca de experiências e aprendizagem. Além disso, a condução do treino em grupo ofereceria a vantagem de alcançar um maior número de participantes do grupo experimental em menor tempo. A literatura internacional já disponibiliza alguns resultados comparativos entre a modalidade coletiva e individual de intervenção (ver Kelly et al., 2014).

No tocante ao número de sessões, os 23 estudos catalogados nesta revisão apresentaram uma grande modificação, com treinos variando de apenas duas
Em relação ao tamanho amostral, os estudos variaram de cinco participantes (Estudo 22) a 112 participantes (Estudo 5), sendo que, dos 23 estudos, 14 apresentaram amostras superiores a 50 participantes $(60,8 \%)$. Sobre o recrutamento dos participantes, vinte estudos realizaram seu recrutamento na comunidade (90,5\%) (Estudos 1, 2, 3, 4, 5, 6, 7, 8, 9, 10, 11, 13, 14, $15,16,17,18,19,21,23)$, e três estudos foram realizados em instituições de longa permanência para idosos $(9,5 \%)$ (Estudos 12, 20, 22). Três estudos incluíram em seu grupo amostral, além de participantes saudáveis, idosos com quadro de comprometimento cognitivo leve (Estudos 5, 15, 22). Em relação a outros quadros de saúde, foi identificado um estudo com idosos hipertensos (Estudo 8) e com diabetes (Estudo 18).

Em relação aos domínios cognitivos treinados, observa-se uma tendência nos estudos nacionais a focarem nas habilidades mnemônicas. Apenas oito estudos puderam ser classificados como multidomínio, ou seja, treinaram habilidades cognitivas diversas $(34,7 \%)$ (Estudos 1, 3, 10, 12, 13, 20, 22, 23), e quinze estudos foram classificados como unimodais $(65,2 \%)$. Deste grupo, um estudo focou em memória de trabalho e 
Pesquisa sistemática da literatura $(\mathrm{n}=33)$

Scielo: 15

PubMed: 7

PsychInfo: 11

Excluídos por duplicação $(\mathrm{n}=6)$

Critérios de exclusão $(\mathrm{n}=8)$

- Ausência de análise estatística de dados $(\mathrm{n}=3)$

- Grupo clínico / reabilitação cognitiva $(n=4)$

- Ausência de exames pré e pós-teste $(\mathrm{n}=1)$

Artigos incluídos na revisão $(\mathrm{n}=19)$

Estudos adicionais incluídos na pesquisa $(\mathrm{n}=4)$

Artigos incluídos para revisão final $(\mathrm{n}=23)$

Artigos substituídos por teses/dissertações ( $\mathrm{n}=4$ )

Figura 1. Processo de seleção dos artigos.

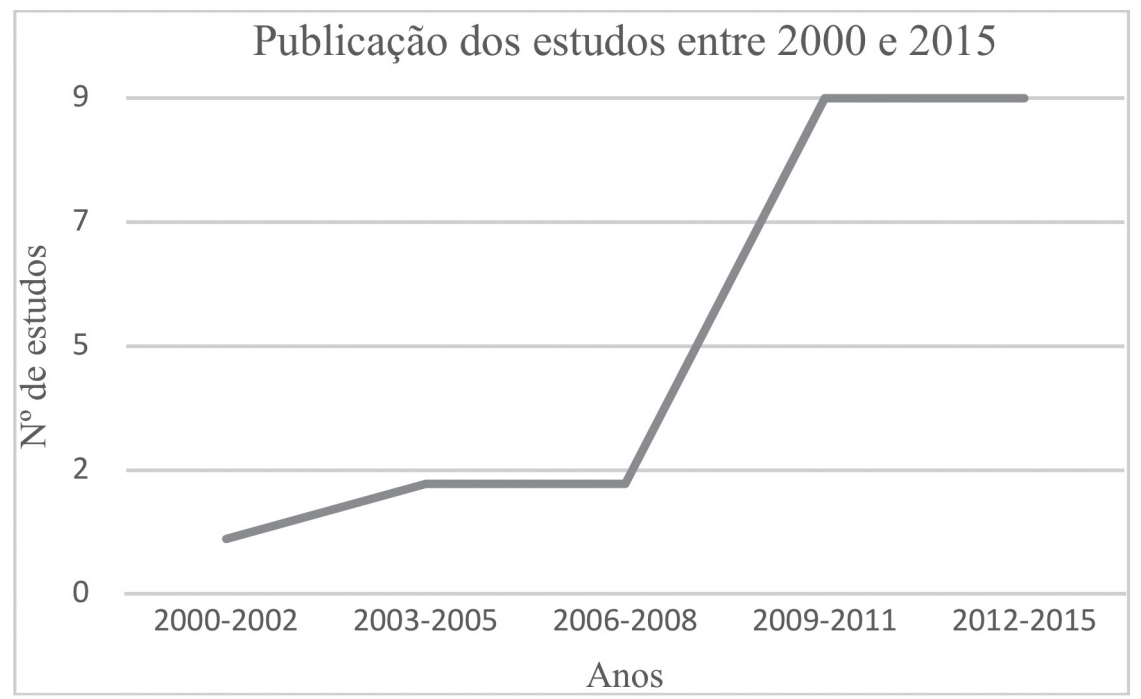

Figura 2. Distribuição temporal de estudos brasileiros sobre treino cognitivo para idosos. 
linguagem para grupos distintos (Estudo 21) e quatorze estudos tiveram como foco principal a memória episódica (Estudos: 2, 4, 5, 6, 7, 8, 9, 11, 14, 15, 16, 17, 18, 19), delimitando uma forte tendência na condução de treinos de memória.

Em relação às medidas cognitivas utilizadas, a análise restringiu-se à bateria pré e pós-intervenção e utilizou como critérios: a) uso de escalas de rastreio cognitivo (ex.: Mini Exame do Estado Mental, Teste Cognitivo Breve); b) uso de medidas subjetivas (escalas de autorrelato); c) uso de escalas cognitivas não restritas a psicólogos; d) uso de testes cognitivos restritos a psicólogos (avaliados e com parecer favorável pelo Conselho Federal de Psicologia). A Tabela 1 faz uma síntese dos resultados a partir desses critérios:

Tabela 1

Medidas Cognitivas Pré e Pós-Intervenção

\begin{tabular}{lc}
\hline Tipo & Estudos \% \\
\hline Rastreio cognitivo & 47,6 \\
Medidas subjetivas & 57,1 \\
Medidas não restritas a psicólogos & 90,5 \\
Medidas restritas a psicólogos & 38,0 \\
\hline
\end{tabular}

No tocante à eficácia dos protocolos de intervenção utilizados para aumento do desempenho cognitivo, esse aspecto foi analisado a partir de resultados significativos $(p<0,05)$ intergrupo, isto é, comparações entre grupo experimental e grupo controle após a intervenção para medidas objetivas. Sendo assim, foram considerados apenas os estudos com inclusão de grupo controle. $\mathrm{O}$ levantamento mostrou que $47,6 \%$ dos estudos reportaram resultados positivos a favor do treino cognitivo. Muitos estudos (52,4\%) reportaram resultados significativos apenas na análise intragrupo, o que não permite inferir por efeitos de ganho do treino.

Finalmente, cabe ressaltar que apenas dois estudos conduziram exames de acompanhamento (follow-up): Aramaki e Yassuda (2011) realizaram um follow-up em 16 participantes, 18 meses após receberam um treino de memória de cinco sessões, porém, sem avaliação de grupo controle; Brum (2012) realizou dois acompanhamentos em uma amostra de 61 participantes, sendo o primeiro um mês após a intervenção e o segundo, seis meses após o treino original, com follow-up também para o grupo controle. Os demais estudos $(90,5 \%)$ não reportaram resultados de acompanhamento.
Essa tendência reflete uma das principais limitações metodológicas da área, também presente nos estudos internacionais, inviabilizando conclusões mais substanciais sobre a manutenção dos efeitos de ganho em longo prazo. A Figura 3 fornece uma síntese ilustrativa das principais características dos estudos nacionais.

Em relação à origem da intervenção - se elaborada para o contexto nacional ou adaptada do contexto internacional - dos 23 estudos analisados, dois não explicitaram essa informação. Do grupo de 21 estudos que reportaram a origem do treino, constatou-se que a maioria dos estudos $(80,9 \%, n=17)$ utilizou intervenções elaboradas para o contexto brasileiro, sendo que apenas quatro estudos procederam à adaptação de protocolos internacionais $(1,2,19,21)$.

\section{Discussão}

Os estudos de intervenção experimental oferecem um caminho mais controlado para investigar a hipótese do enriquecimento cognitivo, pois submetem o indivíduo ao tratamento que, por sua vez está sob controle experimental. Apesar dos problemas metodológicos inerentes a qualquer desenho experimental, como a dificuldade (ou impossibilidade) da randomização completa das condições, existe um reconhecimento na área de que o método ideal de investigação dos efeitos de uma intervenção cognitiva é um ensaio clínico randomizado, no qual um grupo de indivíduos é atribuído ao tratamento experimental (neste caso, o treino cognitivo) e um outro grupo é atribuído a uma atividade de controle, e ambos os grupos são avaliados para determinar os efeitos da intervenção de uma ou mais variáveis de resultado. Salthouse (2006) propõe três características essenciais para os ensaios clínicos. A primeira é a atribuição aleatória de indivíduos para os grupos experimental e controle, para minimizar as influências associadas com as diferenças pré-existentes, tais como nível inicial de habilidade cognitiva e escolarização. A segunda característica é o rigoroso controle do tratamento em relação ao tipo e quantidade de exercício cognitivo. E a terceira característica sugerida é o monitoramento de longo prazo (exames de follow-up) para permitir inferências sobre a taxa de envelhecimento mental e os efeitos de durabilidade da intervenção.

A condução deste estudo de revisão sistemática permitiu traçar um cenário atual das principais tendências metodológicas dos estudos nacionais no campo do treino cognitivo para idosos e como a literatura nacional situa-se no alcance da elucidação da hipótese 


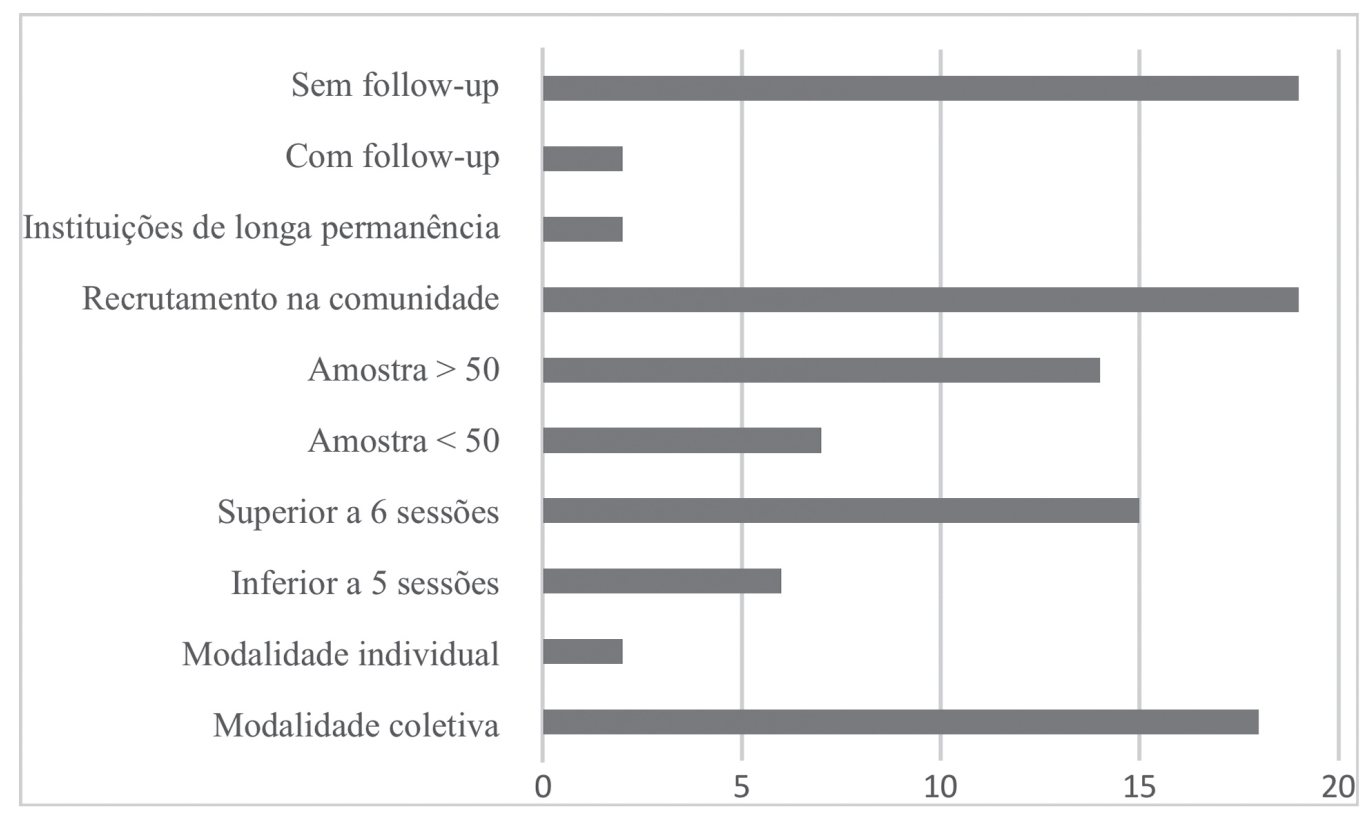

Figura 3. Principais características dos estudos nacionais.

do enriquecimento cognitivo durante a velhice. Nesse sentido, algumas considerações metodológicas se fazem necessárias. A primeira diz respeito à dificuldade de acesso a informações completas dos desenhos de alguns estudos, especialmente sobre a randomização da amostra e à classificação do ensaio (duplo cego ou único cego), problema reportado também em alguns estudos de meta-análise e revisão sistemática internacionais (ex.: Martin et al., 2011; Papp et al., 2009). Observa-se a necessidade de um avanço em relação à consolidação de critérios para divulgação de ensaios clínicos, evitando que sejam reportados de forma incompleta ou inadequada, o que dificulta (ou impossibilita) a realização de estudos de meta-análise e de revisão sistemática. No cenário internacional, muitas iniciativas nesse sentido já estão consolidadas, tais como o Consolidated Standards of Reporting Trials (CONSORT: http://www.consort-statement.org/), que disponibiliza guidelines para a divulgação de ensaios clínicos.

Uma próxima consideração metodológica diz respeito às medidas objetivas utilizadas nas avaliações pré e pós-treino, muitas das quais não estão aprovadas pela comissão consultiva do Conselho Federal de Psicologia, o Satepsi (Sistema de Avaliação de Testes Psicológicos, Conselho Federal de Psicologia - CFP). As medidas cognitivas utilizadas nos estudos nacionais são bastante variadas e a maioria classifica-se como de domínio comum (não restrito a psicólogos). Considerando que o campo das intervenções cognitivas não está restrito à Psicologia, é natural que muitos pesquisadores optem por esse tipo de escala. Porém, no conjunto dos 23 estudos analisados neste artigo, constatou-se que 18 $(78,2 \%)$ tiveram, em sua autoria, pelo menos um psicólogo. Os demais estudos foram conduzidos por profissionais de diversas áreas da saúde (Fonoaudiologia, Enfermagem, Educação Física, Gerontologia). Uma hipótese levantada para explicar esse perfil é a administração relativamente mais simples desse tipo de escala cognitiva, além dos custos financeiros mais baixos. Porém, tais escalas não são submetidas à avaliação e aprovação da comissão consultiva do Conselho Federal de Psicologia (Sistema de Avaliação de Testes Psicológicos: http://satepsi.cfp.org.br/), que avalia com rigor os índices psicométricos das escalas (fontes de evidência de validade e precisão) e a presença de estudos satisfatórios de padronização e normatização para nosso contexto. Utilizar medidas que não passam por tal avaliação antes de serem aprovadas para uso pode fragilizar o processo de inferência sobre os efeitos da intervenção.

Vale ressaltar, também o problema com a utilização de escalas de rastreio cognitivo para diagnóstico de quadros demenciais (ex.: Mini Exame do Estado Mental) como medidas de pré e pós-teste para embasar inferências sobre os efeitos do treino. Tais escalas não detectam aspectos específicos do funcionamento 
mental e acredita-se que devam restringir-se às baterias de triagem dos participantes. Aliado a isso, tem-se as medidas subjetivas e de autorrelato (como medidas de autopercepção, queixas de memória, satisfação e qualidade de vida), utilizadas também para embasar inferências sobre o efeito de generalização dos ganhos do treino para outros contextos (normalmente para o contexto do funcionamento cotidiano). O problema com as medidas subjetivas é que se tratam de entrevistas de autorrelato que podem ser contaminadas com vieses do informante, comprometendo, portanto, sua validade. $\mathrm{O}$ uso das escalas subjetivas também fragiliza o processo de inferência sobre os efeitos da intervenção, mais especificamente os efeitos de generalização. O uso de medidas subjetivas em detrimento de medidas cognitivas objetivas vem sendo criticado na literatura internacional por fragilizar o processo de inferência sobre o impacto dos treinos no desempenho mental dos participantes (ex.: Hertzgov et al., 2009; Shubert, Strobach, \& Karbach, 2014).

Dentre os estudos nacionais revisados que utilizaram medidas objetivas e aprovadas pelo Satepsi, observou-se que quatro estudos incluíram na sua bateria cognitiva o subteste Códigos da Escala Wechsler de Inteligência para Adultos (WAIS-III), um estudo incluiu o subteste Procurar Símbolos (WAIS-III), quatro estudos incluíram os testes Teste Wisconsin de Classificação de Cartas (WCST) e/ou o Instrumento de Avaliação Neuropsicológica Breve (Neupsilin). Diante dos problemas levantados e do número reduzido de estudos que utilizaram medidas cognitivas psicometricamente robustas (apenas 30\%), constata-se que a área sofre com um grave problema de mensuração dos efeitos de intervenção, possivelmente devido a limitações práticas no uso desses instrumentos, que demandam esforços financeiros e de tempo consideráveis em sua administração.

Além do problema relativo aos instrumentos utilizados nas avaliações pré e pós-intervenção, que abrangeu a quase totalidade dos estudos nacionais, destacam-se problemas metodológicos pontuais, tais como:

A) A ausência de um grupo controle em cinco estudos.

B) Utilização de grupos de treino como controles uns dos outros $(n=3)$.

b1. Em um estudo, a amostra foi dividida em dois grupos e cada um treinou uma estratégia mnemônica diferente (categorização e imagem visual), sendo um considerado o controle do outro.

b2. Em um estudo, os grupos de treino em componentes diferentes da memória de trabalho também foram considerados como controles uns dos outros, mas faz-se uma ressalva neste estudo, pois o objetivo principal do autor era testar modelos teóricos por meio da intervenção, o que justifica esse desenho.

b3. Em um estudo, os grupos institucionalizado e não institucionalizado receberam treino e também foram considerados controle um do outro.

C) Recrutamento de participantes provenientes de centros que já trabalham com atividades mentais direcionadas, tornando difícil o isolamento dos efeitos do treino investigado $(n=5)$.

c1. Em dois estudos, os participantes do grupo experimental e controle foram recrutados de oficinas para memória da Universidade Aberta para a Terceira Idade (Universidade de São Paulo - USP);

c2. Em dois estudos, também houve recrutamento dos participantes matriculados no referido centro, mas não foram especificadas as atividades em que estavam engajados. Complementa-se que a Universidade Aberta à Terceira Idade da USP oferece atividades diversas para indivíduos a partir dos 60 anos, tais como: cursar disciplinas regulares oferecidas na universidade e participar de atividades didático-culturais e físicas;

c3. Em um estudo, os participantes foram recrutados de um curso de alfabetização para adultos.

Em relação à eficácia dos estudos, houve uma dificuldade de alcançar conclusões robustas para esse critério nos estudos de revisão, pois nota-se que o campo de pesquisa nacional ainda não dispõe de um 
consenso sobre os critérios para se avaliar a eficácia de um treino. Alguns estudos baseiam-se na observação de diferenças significativas nas médias (ou deltas) entre pré e pós-teste em uma análise intragrupo, outros baseiamse em análises mais confiáveis para esse fim, como a análise de variância e os efeitos de interação entre o momento e o grupo, este último critério predominantemente adotado na literatura internacional (Kelly et al., 2014). Outros, ainda, apoiam-se em critérios como aproximação da significância estatística ou observação das médias de desempenho para inferir sobre os efeitos do treino. Porém, uma avaliação cuidadosa dos resultados reportados permitiu concluir que muitos deles não apresentam resultados que sustentem as conclusões geralmente informadas de eficácia das intervenções. Aliado a isto, há o agravante do uso de medidas de rastreio e de medidas subjetivas (autorrelato) para embasar tais conclusões. De todo modo, o presente artigo concluiu que dez estudos $(47,6 \%)$ reportaram dados que sugerem efeitos de treino para pelo menos uma medida cognitiva objetiva utilizada.

Ainda em relação à eficácia das intervenções, um dos critérios mais importantes nessa análise e que ainda configura-se como ponto de contenção na área é a obtenção de efeitos de durabilidade temporal dos ganhos advindos do treino. De acordo com os estudos analisados neste artigo, observa-se que o cenário nacional encontra-se bastante defasado em relação à investigação desse efeito, comprometendo sobremaneira o alcance de conclusões mais robustas sobre o impacto das intervenções na modificabilidade da estrutura cognitiva. Em relação à investigação dos efeitos de generalização para habilidades não treinadas, é possível observar uma maior preocupação dos estudos analisados com a inclusão, na bateria pré e pós-intervenção, de medidas de habilidades funcionais e de medidas de habilidades cognitivas que não foram alvo da intervenção, permitindo que o campo nacional avance na investigação dos efeitos de generalização e transferência distal (far transfer). Por fim, a investigação dos efeitos de ganho para habilidades treinadas (near transfer) foi a mais comumente reportada nos estudos analisados, o que também permite um avanço nesse aspecto. Porém, esse efeito deve ser considerado com cautela, pois muitos estudos utilizam medidas bastante semelhantes (e por vezes iguais) às tarefas treinadas na intervenção. De todo modo, esse resultado está consoante com o reportado na literatura internacional, em que se observa um maior consenso sobre o impacto positivo dos treinso nas habilidades-alvo da intervenção (Kelly et al., 2014; Law et al., 2014; Simon et al., 2012).

\section{Conclusão}

Finalmente, é importante ressaltar que muitos são os desafios para condução de estudos experimentais com população idosa. Em geral, um dado campo de pesquisa assume algumas recomendações de delineamentos sobre como observar e/ou manipular o fenômeno pertinente à hipótese investigada. Mas os problemas se tornam especialmente difíceis quando se trata da hipótese do enriquecimento cognitivo, pois ela implica que as mudanças na cognição ao longo da vida serão afetadas pelo estilo de vida e comportamentos dos indivíduos, além das dificuldades de se isolar os efeitos do treino de quaisquer outros efeitos. Os problemas metodológicos encontrados na avaliação das hipóteses sobre o desenvolvimento adulto-idoso são vários e vem sendo explicitados por alguns autores (ex.: Hertgzov et al., 2013; Salthouse, 2006). Devido ao desafio de condução de ensaios clínicos e a dificuldade de isolar o papel do treino cognitivo em quaisquer efeitos que podem ser encontrados, Salthouse (2006) concluiu que todas as pesquisas de intervenção experimental na área do envelhecimento cognitivo são baseadas apenas em aproximações do ensaio ideal, sendo que em cada uma falta uma ou mais das características fundamentais para um delineamento experimental.

Assim, é necessário que o campo de pesquisa nacional em treino cognitivo para idosos seja fortalecido em termos de ampliação do número de estudos e se aperfeiçoe seus aspectos metodológicos, buscando a replicação dos ensaios para protocolos já construídos para nosso contexto, assim como à construção de novos protocolos de treino e sua investigação. Outro aspecto de fundamental importância é que a área alcance uma padronização da divulgação dos resultados, de forma a possibilitar, com o acúmulo de evidências, a realização de estudos de meta-análise.

\section{Referências}

Aramaki, F. O., \& Yassuda, M. S. (2011). Cognitive Training based on metamemory and mental images: Follow-up evaluation and booster training effects. Dementia and Neuropsychology, 5(1), 48-53. Recuperado de http://www.demneuropsy.com. br/detalhe_artigo.asp?id $=262$ 
Ball, K., Berch, D. B., Helmers, K. F., Jobe, J. B., Leveck, M. D., Marsiske, M., Morris, J. N., Rebok, G. W., Smith, D. M., Teennstedt, S. L., Unverzagt, F. W., \& Willis, S. L. (2002). Effects of cognitive training interventions with older adults: a randomized controlled trial. JAMA, 288, 2271-2281. Recuperado de http://www.ncbi.nlm.nih.gov/pmc/articles/ PMC2916176/pdf/nihms24855.pdf

Bahar-Fuchs, A., Clare, L., \& Woods, B. (2013). Cognitive training and cognitive rehabilitation for mild to moderate Alzheimer's disease and vascular dementia. The Cochrane Library, vol 6. doi: 10.1002/14651858.CD003260.pub2

Baltes P. B., \& Lindenberger, V. (1998). On the range of cognitive plasticity in old age as a function of experience: 15 years of intervention research. Behavior Therapy, 19, 283-300. Recuperado de http:// library.mpib-berlin.mpg.de/ft/pb/PB_On_the_ Range_1988.pdf

Baltes, P. B., \& Willis, S. L. (1982). Plasticity and enhancement of intellectual functioning in old age. Penn State's Adult Development and Enrichment Project (ADEPT). Em F. I. M. Craik \& S. Trehulo (Eds.). Aging and cognitive processes (353-389). New York: Plenum Press.

Belleville, S (2008). Cognitive training for persons with mild cognitive impairment. InternationalPsychogeriatrics, 20(1), 57-66. doi: 10.1017/S104161020700631X

Brum, P. S. (2012). Treino de memória para idosos saudáveis e com comprometimento cognitivo leve: Beneficios sobre parâmetros cognitivos (Dissertação de mestrado). Universidade de São Paulo, SP. Recuperado de http:// www.teses.usp.br/teses/disponiveis/5/5142/tde27072012-152403/pt-br.php

Buschert, V., Bokde, A. L. W., \& Hampel, H (2010). Cognitive intervention in Alzheimer disease, $\mathrm{Na}$ ture Reviews Neurology, 6(9), 508-517. doi: 10.1038/ nrneurol.2010.113

Carvalho, F C., Neri, A. L., \& Yassuda, M. S. (2010). Treino de memória episódica com ênfase em categorização para idosos sem demência e depressão. Psicologia: Reflexão e Crítica 23(2), 317-323. doi: 10.1590/S0102-79722010000200014

Chariglione, I. P. F (2010). A influência de diferentes tipos de treinos cognitivos na memória de idosos institucionalizados (Dissertação de mestrado). Institutp de Psicologia, Universidade de Brasília, Brasília, DF.
Recuperado de http://repositorio.unb.br/ handle $/ 10482 / 8164$ ? mode $=$ full

Chariglione, I. P. F. (2014). Intervenções cognitivas para o aprimoramento da memória em idosos com envelhecimento cognitivo normal (Tese de doutorado). Instituto de Psicologia, Universidade de Brasília, Brasília, DF. Recuperado de http://repositorio.unb.br/ handle/10482/16137

Clare, L., \& Woods, R. T. (2004). Cognitive training and cognitive rehabilitation for people with early-stage Alzheimer's disease: A review, Neuropsychological Rehabilitation, 14(4), 385-401. doi: 10.1080/09602010443000074

Clare, L., Woods, R. T., Cook, E. D. M., Orrell, M., \& Spector, A (2003). Cognitive rehabilitation and cognitive training for earlystage Alzheimer's disease and vascular dementia, Cochrane Database Systematic Review, 4. doi: 10.1002/14651858.CD003260

Dias, M. S., \& Lima, R. M. (2012). Estimulação cognitiva por meio de atividades físicas em idosas: Examinando uma proposta de intervenção. Revista Brasileira de Geriatria e Gerontologia, 15(2), 325-334. doi: 10.1590/ S1809-98232012000200015

Gates, N. J., Sachdev, P. S., Singh, M. A. F., \& Valenzuela, M (2011). Cognitive and memory training in adults at risk of dementia: A systematic review. BMC Geriatrics, 11(55), 1-14. doi: 10.1186/1471-2318-11-55

Hertzov, C., Kramer, A. F., Wilson, R. S., \& Lindenberger, U. (2008). Enrichment effects on adult cognitive development: Can the functional capacity of older adults be preserved and enhanced? Psychological Science in the Public Interest, 9 (1), 1-65. doi: 10.1111/j.1539-6053.2009.01034.x

Irigaray, T. Q., Filho, I. G., Schneider, R. H. (2010). Efeitos de um treino de atenção, memória e funções executivas na cognição de idosos saudáveis. Psicologia: Reflexão e Crítica, 25(1), 188-202. doi: 10.1590/ S0102-79722012000100023

Irigaray, T. Q., Schneider, R. H., \& Gomes, I. (2004). Efeitos de um treino cognitivo na qualidade de vida e no bem-estar psicológico de idosos. Psicologia: Reflexão e Crítica, 24(4), 810-818. doi: 10.1590/ S0102-79722011000400022

Kelly, M. E., Loughrey, D., Lawlor, B. A., Robertson, I. H., Walsh, C., \& Brennan, S (2014). The impact of cognitive training and mental stimulation on 
cognitive and everyday functioning of healthy older adults: A systematic review and meta-analysis. Ageing Research Reviews, 15, 28-46. doi: 10.1016/j. arr.2014.02.004.

Kramer, A. F., \& Willis, S. (2003). Cognitive plasticity and aging. Em B. Ross (Ed), Psychology of learning and motivation, 43, 267-302. New York: Academic Press.

Lasca, V. B. (2003). Treinamento de memória no envelhecimento normal: Um estudo experimental utilizando a técnica de organização (Tese de doutorado). Universidade Estadual de Campinas, Campinas, SP. Recuperado de: www.bibliotecadigital.unicamp. $\mathrm{br} /$ document $/$ ?down $=$ vtls000308781

Law, L. L. F., Barnett, F., Yau, M. K., \& Gray, M. A. (2014). Effects of combined cognitive and exercise interventions on cognition in older adults with and without cognitive impairment: A systematic review. Ageing Research Review, 15, 61-75. doi: 10.1016/j.arr.2014.02.008

Lima-Silva et al (2011). Treino cognitivo para idosos baseado em estratégias de categorização e cálculos semelhantes a tarefas do cotidiano. Revista Brasileira de Geriatria e Gerontologia, 14(1), 65-74. doi: 10.1590/ S1809-98232011000100008

Lima-Silva, T. B. et al. (2010). Effects of cognitive training based on metamemory and mental images. Dementia and Neuropsychology, 4(2), 114-119. Recuperado de http://www.demneuropsy.com.br/ imageBank/PDF/v4n2a07.pdf

Lima-Silva, T. B., \& Yassuda, M.S. (2011) Treino cognitivo e intervenção psicoeducativa para indivíduos hipertensos: Efeitos na cognição. Psicologia: Reflexão e Crítica, 25(1), 30-40. doi: 10.1590/ S0102-79722012000100005

Martin, M., Clare, L., Altsgassen, A. M., Cameron, M. H., \& Zehnder, F. (2011). Cognition-based interventions for healthy older people and people with mild cognitive impairment. The Cochrane Library, 1, 1-51. doi: 10.1002/14651858.CD006220.pub2.

Neri, A. L (1993). Qualidade de vida e idade madura. Campinas: Ed. Papirus. 1993.

Olchik, M. R. (2008). Treino de memória: um novo aprender no envelhecimento (Tese de doutorado). Universidade Federal do Rio Grande do Sul, Porto Alegre,
RS). Recuperado de http://www.lume.ufrgs.br/ handle/10183/13489

Olchik, M. R., Mattos, D., Maineri, N. S., Oliveira, A., \& Yassuda, M. S. (2012). Revisão: Efeitos do treino de memória em idosos normais e em idosos com comprometimento cognitivo leve. Temas em Psicologia, 20(1), 235-245. Recuperado de http://pepsic.bvsalud.org/scielo. php?pid $=$ S1413389X2012000100017\&script $=$ sci_arttext

Oliveira, T. C., G., Soares, F. C., Macedo, L. D. D., Wanderley, D. L., Diniz, P., Bento-Torres, N. V. O., \& Picanço-Diniz, C. W. (2014). Beneficial effects of multisensory and cognitive stimulation on age-related cognitive decline in long-term-care institutions. Clinical Interventions in Aging, 14(9), 309321. doi: 10.2147/CIA.S54383

Papp, K. V., Stephen, J. W., \& Peter, J. S. (2009). Immediate and delayed of cognitive interventions in healthy elderly: A review of current literature and future directions. Al₹heimer's and Dementia, 5, p 5060. doi: 10.1016/j.jalz.2008.10.008

Paulo, D. L. V., \& Yassuda, M. S. (2012). Elderly individuals with diabetes: Adding cognitive training to psychoeducational intervention. Educational Gerontology, 38(4), 257-270. doi: 10.1080/03601277.2010.544575

Reijnders, J., Van Heugten, C., Van Boxtel, M. (2013). Cognitive interventions in healthy older adults and people with mild cognitive impairment: A systematic review. Aging Research Review, 12, p. 263-275. doi: 10.1016/j.arr.2012.07.003

Salthouse, T. A. (2006). Mental exercise and mental aging: Evaluating the validity of the "use it or lose it" hypothesis. Perspectives on Psychological Science, 1, 6887. doi: 10.1111/j.1745-6916.2006.00005.x

Santos, I. B. (2010). Oficinas de estimulação cognitiva em idosos analfabetos e com transtorno cognitivo leve (Dissertação de mestrado). Universidade Católica de Brasilia, Brasília, DF.Recuperado de: www.bdtd.ucb.br/ tede/tde_busca/arquivo.php?codArquivo=1231

Schaie, K.W., \& Willis, L. (1986) Can decline in adult intellectual functioning be reversed? Development Psychology. 22(2), 223-232. doi: 10.1037/0012-1649.22.2.223 
Silva, H. S., \& Yassuda, M. S. (2009). Memory Training for older adults with low education: Mental images versus categorization. Educational Gerontology, 35(10), 890-905. doi: 10.1080/03601270902782487

Simon, S. S., Yokomizo, J. E., \& Bottino, C. M. C. (2012). Cognitive intervention in amnestic mild cognitive impairment: A systematic review. Neuroscience and Behavioral Reviews, 36, 1163-1178. doi: 10.1016/j. neubiorev.2012.01.007

Soares, Z. F., Santana, E. F., \& Rabelo, D. F. (2015). Iniciação à informática associada ao treino cognitivo em idosos. Revista Ciência em Extensão, 11(3), 155-167. Recuperado de http://ojs.unesp.br/index.php/revista_proex/article/view/1112

Shubert, T., Strobach, T., \& Karbach, J. (2014). New directions in cognitive training: On methods, transfer and applications. Psychological Research, 78, 749755. doi: 10.1007/s00426-014-0619-8.

Tardif, S., \& Simard, M (2011). Cognitive stimulation programs in healthy elderly: A review. International Journal of Alzheimer's Disease, may/2011, 1-13. doi: 10.4061/2011/378934

Tavares, P. N., Schimidt, J. H., \& Witter, C (2015). Efeitos de um programa de intervenção no desempenho cognitivo e sintomatologia depressiva em idosos institucionalizados. Revista Kairós Gerontologia, 18(2), 103-123. Recuperado de http://revistas.pucsp.br/index.php/kairos/article/view/26316

Teixeira-Fabrício et al. (2012). Treino cognitivo em adultos maduros e idosos: Impacto de estratégias segundo faixas de escolaridade. Psico-USF, 17(1), 85-95. doi: 10.1590/S1413-82712012000100010

Valenzuela, M., \& Sachdev, P. (2009). Can cognitive exercise prevent the onset dementia? Systematic review of randomized clinical trials with longitudinal follow-up. American Journal of Geriatric Psychiatry, 17(3), 179-187. doi: 10.1097/JGP.0b013e3181953b57
Verhaeghen, P., Marcoen, A., \& Goossens, L. (1992). Improving memory performance in the aged trough mnemonic training: A meta-analytic study. Psychology and Aging, 7(2), 242-251. Recuperado de http://content.apa.org/journals/pag/7/2/242

Willis, S. L., \& Nesselroade, C. S. (1990) Long term effects of fluid ability training in old-old age. Developmental Psychology; 26,905-910. Recuperado de http://psycnet.apa.org/index.cfm?fa=buy. option'ToBuy\&id=1991-07074-001

Willis, S. L., Blieszener, R., \& Baltes, P. B. (1981). Intellectual training research in aging: modification of performance on the fluid ability of figural relations. Journal of Educational Psychology, 73(1), 41-50. Recuperado de http://psycnet.apa.org/index. cfm? $\mathrm{fa}=$ buy.option ToBuy\&id $=1981-07746-001$

Wood, G. M. O. (2000). Efeitos do nivel de autoeficácia cognitiva percebida e de programas de treinamento cognitivo sobre a capacidade de memória de trabalho de indivíduos idosos (Dissertação de mestrado). Universidade Federal de Minas Gerais, Belo Horizonte, MG.

Yassuda, M. S., Batistoni, S. S. T., Fortes, A. G., \& Neri, A. L. (2006). Treino de memória no idoso saudável: Benefícios e mecanismos. Psicologia: Reflexão e Crítica, 19(3), 470-481. doi: 10.1590/ S0102-79722006000300016

Zimmermann, N., Netto, T. M., Amodeo, M. T., Ska, B., \& Fonseca, R. P (2014). Working memory training and poetry-based stimulation programs: Are the differences in cognitive outcomes in healthy older adults? Neuro Rehabilitation, 35(1), 159-170. doi: 10.3233/NRE-141104

Recebido em: 13/10/2015

Reformulado em: 11/04/2016

Aceito em: 18/04/2016 
Sobre as autoras:

Mariana Teles Santos é psicóloga, mestre em Psicologia do Desenvolvimento Humano pela Universidade Federal de Minas Gerais (UFMG), doutora em Psicologia do Desenvolvimento Humano (UFMG), com pós-doutorado em Psicologia Cognitiva e Comportamento Social pela Universidade Federal de São Carlos, e professora adjunta do Instituto Multidisciplinar em Saúde da Universidade Federal da Bahia.

E-mail: marianatsantos84@gmail.com

Carmen Flores-Mendoza é psicóloga, mestre em Psicologia Clínica pela PUC -Campinas, doutora pela Universidade de São Paulo em Psicologia da Aprendizagem e Desenvolvimento Humano, com pós-doutorado na University of California, at Davis em Metodologia Quantitativa. É professora do Departamento de Psicologia e do PPG em Psicologia e Neurociências da UFMG e cofundadora do Laboratório de Avaliação das Diferenças Individuais.

E-mail: carmencita@fafich.ufmg.br

Contato com as autoras:

Rua Manoel Januário de Andrade, 414

Recreio, Vitória da Conquista-BA, Brasil

CEP: 45020-540 
